\title{
Relationship between $A D I P O Q$ gene, circulating high molecular weight adiponectin and albuminuria in individuals with normal kidney function: evidence from a family-based study
}

\author{
C. Menzaghi • S. De Cosmo • M. Copetti • L. Salvemini • \\ C. De Bonis • D. Mangiacotti • G. Fini • F. Pellegrini • \\ V. Trischitta
}

Received: 11 September 2010 / Accepted: 14 December 2010 /Published online: 13 January 2011

(C) Springer-Verlag 2011

\begin{abstract}
Aims/hypothesis Insulin resistance is associated with reduced serum adiponectin and increased albuminuria levels. Thus, one would anticipate an inverse relationship between circulating adiponectin and albuminuria. However, several studies have described a 'paradoxical' elevation of serum adiponectin in patients with elevated albuminuria. These findings may have been confounded by the presence of diseases and related treatments known to affect circulating adiponectin and albuminuria. We therefore studied the
\end{abstract}

C. Menzaghi and S. De Cosmo contributed equally to this study.

Electronic supplementary material The online version of this article (doi:10.1007/s00125-010-2037-9) contains supplementary material, which is available to authorised users.

C. Menzaghi - L. Salvemini - C. De Bonis • D. Mangiacotti • G. Fini $\cdot$ V. Trischitta $(\square)$

Research Unit of Diabetes and Endocrine Disease,

IRCCS Casa Sollievo della Sofferenza,

Viale Padre Pio,

71013, San Giovanni Rotondo, Italy

e-mail: v.trischitta@operapadrepio.it

\section{S. De Cosmo}

Unit of Endocrinology, IRCCS Casa Sollievo della Sofferenza,

San Giovanni Rotondo, Italy

M. Copetti $\cdot$ F. Pellegrini

Unit of Biostatistics, IRCCS Casa Sollievo della Sofferenza,

San Giovanni Rotondo, Italy

V. Trischitta

Department of Experimental Medicine,

Sapienza University of Rome,

Rome, Italy relationship between circulating adiponectin and albuminuria in the absence of such confounders.

Methods To this purpose, the relationship between adiponectin isoforms and albumin:creatinine ratio (ACR) was investigated in a family-based sample of 634 non-diabetic untreated white individuals with normal kidney function. We also investigated whether the two variables share a common genetic background and addressed the specific role of the gene encoding adiponectin on that background by genotyping several $A D I P O Q$ single nucleotide polymorphisms (SNPs).

Results ACR was directly associated with high molecular weight (HMW) adiponectin isoform $(p=0.024)$. The two variables shared some genetic correlation $\left(\rho_{\mathrm{g}}=0.38\right.$, $p=0.04)$. ADIPOQ promoter SNP rs 17300539 was associated with HMW adiponectin $\left(p=4.8 \times 10^{-5}\right)$ and ACR $(p=0.0027)$. The genetic correlation between HMW adiponectin and ACR was no longer significant when SNP rs17300539 was added to the model, thus reinforcing the role of this SNP in determining both traits.

Conclusions/interpretation Our study shows a positive, independent correlation between HWM adiponectin and ACR. $A D I P O Q$ variability is associated with HMW adiponectin and ACR, and explains some of the common genetic background shared by these traits, thus suggesting that $A D I P O Q$ and $\mathrm{HMW}$ adiponectin modulate albuminuria levels.

Keywords Adiponectin HMW - $A D I P O Q$ gene ·

Albuminuria $\cdot$ Mendelian randomisation
Abbreviations
ACR Albumin/creatinine ratio
HMW High molecular weight 
HOMA $_{\mathrm{IR}}$ HOMA insulin resistance index

LMW Low molecular weight

MMW Medium molecular weight

SNP Single nucleotide polymorphism

SOLAR Sequential oligogenic linkage analysis routines

UAE Urinary albumin excretion

\section{Introduction}

Increased urinary albumin excretion (UAE) rate in the range of micro- or macro-albuminuria is a marker of kidney disease [1], and a strong and established predictor of morbidity and mortality rates for cardiovascular disease $[2,3]$.

Adiponectin, a hormone exclusively secreted by adipocytes, circulates abundantly in the bloodstream, as low, medium and high molecular weight (LMW, MMW and HMW) multimers [4, 5] and has well-recognised antiatherogenic, anti-inflammatory and insulin-sensitising properties $[4,6]$. Studies in humans have persistently reported that insulin resistance and related clinical outcomes are associated with lower adiponectin concentrations [7-9] as well as with increased UAE [10, 11]. Based upon these well established associations, one would expect an inverse relationship between circulating adiponectin and albuminuria. However, several studies have described a 'paradoxical' elevation of serum adiponectin concentrations in patients with elevated UAE [12-18]. The reasons for such unexpected findings are unknown. Unfortunately, results of most studies addressing this issue may have been confounded by the presence of diseases (i.e. diabetes and diabetic or non-diabetic kidney disease) and/or treatments (i.e. statins, renin-angiotensin system inhibitors and thiazolinediones) known to affect circulating adiponectin and UAE levels [19-24].

The aim of the present study was to investigate the relationship between circulating adiponectin and UAE in the absence of the above-mentioned confounders.

To this purpose, a family-based sample of 634 nondiabetic, untreated white individuals with normal kidney function was investigated for association between serum adiponectin isoforms and UAE, expressed as albumin: creatinine ratio (ACR). We also addressed the issue of whether or not circulating adiponectin isoforms share a common genetic background with ACR and, if so, if it is partly explained by $A D I P O Q$ gene variability. To this latter purpose, we genotyped single nucleotide polymorphisms (SNPs) in the $A D I P O Q$ gene or their proxies (as indicated by $r^{2}>0.9$ ) (see Electronic supplementary material [ESM] Table 1), which have been consistently associated with adiponectin levels [25-28].

\section{Methods}

Study population We recruited 634 non-diabetic individuals with normal kidney function (i.e. cystatin C-GFR $\geq 60 \mathrm{ml} / \mathrm{min}$ ) from 233 families living in the Gargano area of Italy (an homogeneous geographical area in central-east Italy) [29] and examined them as previously described [30-32]. All study participants were not being treated with medications known to interfere with glucose homeostasis, lipid profile and blood pressure, or with those known to modulate adiponectin and ACR levels. The study and informed consent procedures were approved by the local Research Committee.

Measurements Serum HMW, MMW and LMW adiponectin concentrations were measured by ELISA (Alpco, Salem, $\mathrm{NH}$, USA) as previously described [32].

Human serum cystatin $\mathrm{C}$ was assayed in duplicates by ELISA (BioVendor, Modrice, Czech Republic). The assay covered the range from 0.2 to $10.0 \mathrm{mg} / 1$ in serum samples diluted 1:400. The intra- and inter-assay coefficients of variation were $<4.5$ and $<4.7$, respectively.

Urinary albumin and creatinine concentrations were determined the same morning of the clinical examination on an early morning first-void sterile urine sample by the nephelometric method (Nephelometer Analyser; Behring, Germany) and the Jaffe's reaction-rate method (737 Autoanalyser; Hitachi, Tokyo, Japan), respectively. Elevated UAE was diagnosed if the ACR was $\geq 2.5 \mathrm{mg} / \mathrm{mmol}$ in men and $\geq 3.5 \mathrm{mg} / \mathrm{mmol}$ in women.

Estimated GFR was calculated by the reciprocal of serum cystatin $C$ expressed in $\mathrm{mg} / \mathrm{l}$ multiplied by 100 and defined as cystatin C-GFR [33].

The metabolic syndrome score was calculated for each study participant summing the number of individual components of the syndrome, according to ATP III criteria, as follows: waist circumference $>102 \mathrm{~cm}$ for men and $>88 \mathrm{~cm}$ for women; systolic blood pressure $\geq 130 \mathrm{mmHg}$ or diastolic blood pressure $\geq 85 \mathrm{mmHg}$; serum HDLcholesterol $<0.9 \mathrm{mmol} / \mathrm{l}$ for men and $<1.0 \mathrm{mmol} / \mathrm{l}$ for women; serum triacylglycerol levels $\geq 1.7 \mathrm{mmol} / \mathrm{l}$; and venous plasma glucose $\geq 6.1 \mathrm{mmol} / 1$ [31]. The HOMA insulin resistance index $\left(\mathrm{HOMA}_{\mathrm{IR}}\right)$ was calculated as fasting serum insulin $(\mathrm{pmol} / \mathrm{l}) \times$ fasting plasma glucose $(\mathrm{mmol} / \mathrm{l}) / 135[31,32]$.

Genotyping SNPs rs17300539, rs182052, rs17366568, rs1501299, and rs6773957 in the ADIPOQ gene were selected because of their previous association with adiponectin circulating levels (see ESM Table 1) and genotyped by Taqman SNP allelic discrimination technique using an device (ABI 7000; Applied Biosystems, CA, USA). Call rate and concordance rate were $>98 \%$ and $>99 \%$, respectively. Of 
634 study individuals, genotypes were available for 621 persons for rs 17300539 , for 624 persons for rs 182052 and for rs17366568, for 623 persons for rs1501299, and for 614 persons for rs6773957. All SNPs were in Hardy-Weinberg equilibrium $(p>0.05)$.

Statistical analysis Data are summarised as means $\pm \mathrm{SD}$ or median (range). Because of skewness for ACR and adiponectin isoforms, logarithmic and square root transformations were performed, respectively, before further analyses.

To determine the contribution of genetic factors to ACR, the Sequential Oligogenic Linkage Analysis Routines (SOLAR) software package (Version 4.1.7) was used [34]. SOLAR performs a variance components analysis of family data that decomposes the total variance of the phenotypes into components that are due to genetic effects (i.e. polygenic, additive genetic variance), measured covariates and random environmental effects (i.e. measured environmental factors and random unmeasured factors). The relative contribution of genetic factors is then estimated by heritability $\left(h^{2}\right)$, defined as the ratio of the genetic variance component to the residual (after removal of covariates) phenotypic variance. Heritability estimates obtained in this way also include any environmental contributions to similarities in adjusted values between relatives. To assess phenotypic correlations between ACR, insulin resistance-related traits and adiponectin isoforms, we used a mixed effects model by SOLAR that includes fixed covariate effects. This method is able to account for the dependence of the family data and provide a more stringent $p$ value. To evaluate the contribution of the $A D I P O Q$ genotypes to ACR variance and test the associations between each trait and each SNP, a linear mixed effects model implemented in SOLAR to account for within-family correlations was performed. Each SNP was included in a model as a fixed effect with additive coding. Sex, age, age squared, smoking habits and physical exercise were first used as covariates when assessing ACR heritability; then also BMI was added to the model. Results were reported as linear model coefficients along with their standard errors and $p$ values $(\beta \pm \mathrm{SE}, p)$. Bivariate analyses were conducted to partition the phenotypic correlation between two traits $(\rho p)$ into genetic $(\rho g)$ and environmental ( $\rho$ e) correlations [34]. Evidence of pleiotropy (i.e. a common set of genes influencing more than one trait) is indicated by a genetic correlation that is significantly different from zero.

A Mendelian randomisation approach [35] was followed to determine whether the association between polymorphisms of ADIPOQ SNP rs17300539 and ACR might be mediated by HMW adiponectin levels. Hierarchical linear models were used to estimate the effect size (i.e. $\beta$ ) per minor allele of $A D I P O Q$ SNP rs17300539 polymorphisms for HMW adiponectin levels $(\beta 1)$ and for ACR ( $\beta 2)$, and the effect size of HMW adiponectin levels for ACR $(\beta 3)$ in the latter two, also including age, sex and BMI as confounders. An unstructured covariance matrix was used to account for familiarity. Mendelian randomisation holds if $\beta 2$ and $\beta 3$ are not statistically different. To test such difference, we treated $\beta 2$ and $\beta 3$ as a two-dimensional variable and introduced a bivariate hierarchical linear model $[36,37]$, which allowed us to simultaneously estimate both adjusted models (ACR vs SNP and ACR vs HWM). We contrasted the two $\beta \mathrm{s}$ at issue within such a framework, which allowed us to account for potential betweenoutcomes correlation.

Power calculation Considering the observed ACR logarithm and the HMW square root standard deviations and accounting for familiarity, our sample size of 634 participants was able to detect a $\beta$ regression coefficient of 0.0325 with a power of $80 \%$ and a $\beta$ regression coefficient of 0.0375 with a power of $90 \%$, at a significance level (alpha) of 0.05 .

\section{Results}

Clinical characteristics of study participants are shown in Table 1.

Among the 634 individuals, the median ACR was $0.53 \mathrm{mg} / \mathrm{mmol}$ (range $0.06-12.4$ ). As per selection criterion, all had cystatin C-GFR $>60 \mathrm{ml} / \mathrm{min}$ (median 120.3, range 60.2-253.8; Table 1).

After adjusting for age, age squared, sex, smoking habits and physical exercise, ACR levels were positively associated with BMI $(\beta=0.008 \pm 0.002, p=0.006)$, waist circumference $(\beta=0.0036 \pm 0.0012, p=0.003)$, diastolic blood pressure $(\beta=0.004 \pm 0.002, p=0.040), \mathrm{HOMA}_{\mathrm{IR}}$ $(\beta=0.13 \pm 0.05, p=0.009)$ and the metabolic syndrome score $(\beta=0.04 \pm 0.01, p=0.026)$. In addition, ACR was directly associated with HMW $(\beta=0.045 \pm 0.02, p=0.024)$, but not with MMW $(\beta=-0.0082 \pm 0.02, p=0.68)$ and LMW ( $\beta=0.03 \pm 0.02, p=0.13)$ adiponectin levels. Adjustment for BMI slightly increased the strength of this association $(\beta=0.06 \pm 0.02, p=0.003)$ (Table 2). Given that cystatin $\mathrm{C}$-GFR in this sample was inversely associated with ACR $(\beta=-1.79 \pm 0.88, p=0.042)$ and HMW adiponectin $(\beta=-6.17 \pm 2.20, p=0.005)$, it was added as an additional covariate to the multivariate model, with virtually no changes observed in the association between ACR and HMW $(\beta=0.06 \pm 0.02, p=0.003)$.

A total of 24 individuals (3.8\% of the whole sample) had elevated ACR. Exclusion of these individuals did not change the results obtained (data not shown). 
Table 1 Clinical characteristics of 634 non-diabetic individuals from 233 families

\begin{tabular}{|c|c|c|}
\hline Characteristic & Mean \pm SD & Median (range) \\
\hline Men/women & $244 / 390$ & \\
\hline Age (years) & $40.3 \pm 14.5$ & $40.0(16-82)$ \\
\hline BMI $\left(\mathrm{kg} / \mathrm{m}^{2}\right)$ & $26.3 \pm 4.7$ & $25.5(17.1-48.2)$ \\
\hline Waist circumference $(\mathrm{cm})$ & $84.7 \pm 12.6$ & $84.0(50-126.0)$ \\
\hline Obese $(\%)$ & 24.3 & \\
\hline Overweight (\%) & 13.3 & \\
\hline Systolic BP (mmHg) & $116.8 \pm 14.9$ & $115.0(80-180)$ \\
\hline Disastolic BP (mmHg) & $77.1 \pm 9.0$ & $80.0(50-112)$ \\
\hline Hypertensive (\%) & 22.1 & \\
\hline Fasting blood glucose (mmol/l) & $5.0 \pm 0.57$ & $4.89(3.20-6.99)$ \\
\hline Insulin (pmol/l) & $55.6 \pm 31.9$ & $49.3(12.5-333.4)$ \\
\hline $\mathrm{HOMA}_{\mathrm{IR}}$ & $1.8 \pm 1.1$ & $1.54(0.36-10.0)$ \\
\hline Triacylglycerol (mmol/1) & $1.1 \pm 0.75$ & $0.9(0.3-5.9)$ \\
\hline HDL-cholesterol (mmol/l) & $1.4 \pm 0.3$ & $1.3(0.5-3.1)$ \\
\hline Metabolic syndrome ( $\%$ affected) & 8.4 & \\
\hline Cystatin C-GFR (ml/min) & $128.7 \pm 56.4$ & $120.3(60.2-253.8)$ \\
\hline Cystatin C (mg/l) & $0.91 \pm 0.36$ & $0.83(0.28-2.20)$ \\
\hline ACR (mg/mmol) & $0.9 \pm 1.16$ & $0.53(0.06-12.4)$ \\
\hline HMW adiponectin $(\mu \mathrm{g} / \mathrm{ml})$ & $4.3 \pm 2.9$ & $3.6(0.02-24.1)$ \\
\hline MMW adiponectin $(\mu \mathrm{g} / \mathrm{ml})$ & $1.6 \pm 1.5$ & $1.2(0.01-11.6)$ \\
\hline LMW adiponectin $(\mu \mathrm{g} / \mathrm{ml})$ & $2.1 \pm 1.8$ & $1.7(0.01-13.5)$ \\
\hline
\end{tabular}

The overall effect of genetic factors on ACR was investigated by variance component analysis. After adjusting for age, age squared, sex, smoking habits and physical exercise, ACR and HMW adiponectin (the latter as previously described [32]) were moderately $\left(h^{2}=0.20 \pm 0.09, p=0.006\right)$ and highly $\left(h^{2}=0.79 \pm 0.06, p=1.0 \times 10^{-13}\right)$ heritable, respectively. A significant positive genetic correlation $\left(\rho_{\mathrm{g}}\right)$ was observed between the two variables $\left(\rho_{\mathrm{g}}=0.38, p=0.04\right)$.

Among the five $A D I P O Q$ SNPs genotyped, only rs 17300539 , located in the promoter region, was significantly

Table 2 Association of serum adiponectin isoforms and ACR in 634 non-diabetic individuals from 233 families

\begin{tabular}{lcc}
\hline Adiponectin isoform & \multicolumn{1}{l}{ ACR $(\mathrm{mg} / \mathrm{mmol})$} & \\
\cline { 2 - 3 } & $\beta \pm \mathrm{SE}$ & $p$ value \\
\hline HMW $(\mu \mathrm{g} / \mathrm{ml})$ & $0.045 \pm 0.02$ & 0.024 \\
HMW $(\mu \mathrm{g} / \mathrm{ml})^{\mathrm{a}}$ & $0.06 \pm 0.02$ & 0.003 \\
MMW $(\mu \mathrm{g} / \mathrm{ml})$ & $-0.0082 \pm 0.02$ & 0.68 \\
LMW $(\mu \mathrm{g} / \mathrm{ml})$ & $0.03 \pm 0.02$ & 0.13 \\
\hline
\end{tabular}

The linear $\beta$ coefficients represent the change in ACR levels for 1 unit increase in the predictor; all analyses were adjusted for age, age squared, sex, smoking habit and physical exercise

${ }^{a}$ Adjusted for age, age squared, sex, smoking habit, physical exercise and BMI associated with ACR ( $p=0.0027$; Table 3 ) and accounted for some proportion of its variance (i.e. $4 \%$ ). This association also remained significant in a model comprising all five SNPs $(p=0.035)$. As previously reported, rs17300539 is also strongly associated with HMW adiponectin $(\beta=0.26 \pm 0.064$, $p=4.8 \times 10^{-5}$ ) and explains almost $10 \%$ of its variance in our sample [32].

The genetic correlation described above between HMW adiponectin and ACR was no longer significant $(p=0.07)$, when SNP rs17300539 was added to the model, thus reinforcing the role of this SNP in partly determining both traits.

Finally, the Mendelian randomisation approach, which was used to investigate whether a cause-effect relationship operates in sustaining the association we observed between HMW and ACR, showed that changes of ACR (i.e. $\beta$ values of hierarchical linear models) that were attributable either to SNP rs17300539 or to HMW adiponectin differences secondary to this SNP were not significantly different $(p=0.20$; Fig. 1). In agreement with this finding, in an age-, sex- and BMI-adjusted linear regression model with SNP rs17300539 and HMW adiponectin as predictors, HWM adiponectin, but not SNP rs17300539, was significantly associated with ACR $(\beta \pm \mathrm{SE}=0.039 \pm 0.012, p=0.0016$ and $0.086 \pm 0.064, p=0.18$, respectively).

\section{Discussion}

Most [12-18], although not all [38-40] previous studies have reported a direct correlation between circulating adiponectin and UAE rate. This finding was somewhat 'unexpected' if insulin resistance was regarded as the common soil of both variables, and pointed to the role of confounding factors, including diabetes, kidney disease and related treatments, possibly affecting previous studies [12-18]. Our study reports a positive and independent correlation between HMW adiponectin and ACR in a homogeneous population of untreated individuals with normal kidney function. In contrast, no correlation between ACR and MMW and/or LMW adiponectin was found. HMW adiponectin is the most biologically active isoform [5]. In addition, HMW, but not MMW and LMW, was associated with insulin resistance in the sample we analysed here [32]. So, although opposite results have been reported in patients with type 2 diabetes [18], one can hypothesise that the lack of correlation between ACR and MMW and LMW reflects the trivial role these two adiponectin isoforms are likely to play in insulin resistance.

To the best of our knowledge, our study also shows, for the first time, that HMW adiponectin and ACR share a common genetic background, which is partially explained by SNP rs17300539, located in the promoter region of the 
Table 3 ACR levels according to $A D I P O Q$ SNPs in 634 non-diabetic individuals from 233 families

Values are reported as mean $\pm \mathrm{SD}$

All analyses adjusted for age, age squared, sex, smoking habit, physical exercise and BMI

\begin{tabular}{llllllll}
\hline ACR per SNP & \multicolumn{2}{l}{ Participants per allele } & & & \\
\cline { 2 - 7 } & Allele & $n$ & Allele & $n$ & Allele & $n$ & $p$ value \\
\hline rs17300539 & GG & 496 & GA & 119 & AA & 6 & \\
ACR $(\mathrm{mg} / \mathrm{mmol})$ & $0.84 \pm 1.15$ & & $0.98 \pm 1.09$ & & $1.72 \pm 3.34$ & & 0.0027 \\
rs182052 & GG & \multirow{2}{*}{316} & GA & 269 & AA & 39 & \\
ACR $(\mathrm{mg} / \mathrm{mmol})$ & $0.85 \pm 1.20$ & & $0.90 \pm 1.20$ & & $0.94 \pm 1.39$ & & 0.50 \\
rs17366568 & GG & 506 & GA & 107 & AA & 11 & \\
ACR $(\mathrm{mg} / \mathrm{mmol})$ & $0.88 \pm 1.20$ & & $0.85 \pm 1.11$ & & $1.15 \pm 1.37$ & & 0.76 \\
rs1501299 & GG & 300 & GT & 259 & TT & 64 & \\
ACR $(\mathrm{mg} / \mathrm{mmol})$ & $0.90 \pm 1.30$ & & $0.85 \pm 1.03$ & & $0.86 \pm 1.06$ & & 0.33 \\
rs6773957 & GG & 157 & GA & 307 & AA & 150 & \\
ACR $(\mathrm{mg} / \mathrm{mmol})$ & $0.83 \pm 0.96$ & & $0.92 \pm 1.32$ & & $0.88 \pm 1.14$ & & 0.82 \\
\hline
\end{tabular}

$A D I P O Q$ gene. Overall, these data confirm the previously observed positive correlation between adiponectin serum concentration and UAE rate, and suggest an underlying cause-effect relationship, with HMW adiponectin (and the $A D I P O Q$ gene) modulating, at least partly, the level of albuminuria. In line with our present findings, SNP rs17300539 has been associated with increased UAE in previous studies $[18,41]$. To test this intriguing hypothesis more thoroughly, we used Mendelian randomisation, which draws on genetic data from cross-sectional association studies to infer causality between phenotypic exposure and outcome of interest [35]. Observed and expected $\beta$ coefficient estimates between HMW adiponectin and ACR levels were not significantly different, thus leaving the possibility open that the biology underlying their association is based, at least partly, upon the role that $A D I P O Q$ promoter SNP rs17300539 plays in increasing adiponectin serum concentrations [42, 43], which, in turn, increase ACR levels. We acknowledge that although nominal statistical significance was not reached, the observed and expected $\beta$-coefficients were quite different in size, with

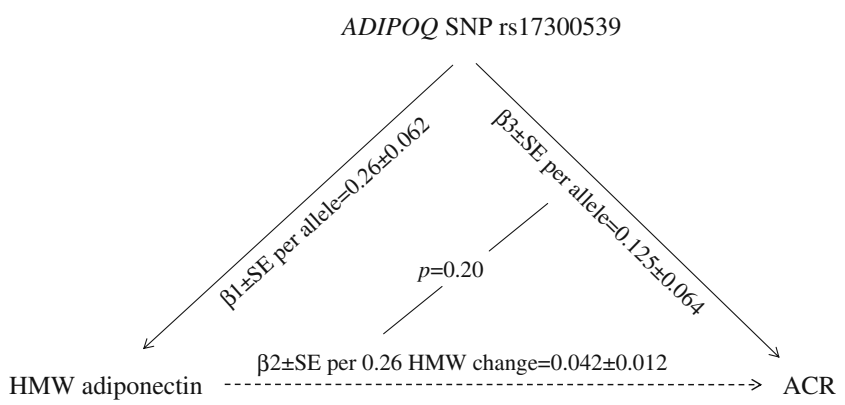

Fig. 1 Results of Mendelian randomisation. $\beta 1$, effect size of association between each minor allele of rs17300539 and HMW adiponectin levels; $\beta 2$, effect size of association between each minor allele of SNP rs17300539 and ACR; $\beta 3$, effect size of association between change of HMW adiponectin levels due to rs17300539 and ACR the observed value being three-times higher than the expected one. So, although our study had $90 \%$ power to detect the observed difference between $\beta$-coefficients as significant at a $\mathrm{p}$ value of 0.05 , and although its familybased design protects against population stratification, thus minimising the risk of false results (type I and II errors) [44], considerable caution is needed when inferring causality from our cross-sectional observation. The difference in size between $\beta$-estimates may be explained by a strong linkage disequilibrium between SNP rs17300539, which modulates HMW adiponectin, and other genetic variant(s) directly modulating ACR. This possibility is supported by several linkage studies pointing to the $3 \mathrm{q} 27$ region (i.e. the same as the $A D I P O Q$ locus) as a candidate region for albuminuria [33, 45-47]. Finally, we cannot rule out the possibility that the positive association between serum HMW adiponectin and ACR levels, even in this normal range of albuminuria, may be due, at least partly, to a homeostatic protective mechanism aimed at counteracting renal and cardiovascular damage. In fact, it has been previously reported that conditions of advanced kidney damage are associated with elevated adiponectin levels [48, 49] and that among patients with end-stage renal disease, adiponectin levels are significantly and inversely related to cardio-metabolic risk factors [49]. The possibility that adiponectin exerts a protective role in patients with renal dysfunction is further supported by the observation that among these patients cardiac events are less frequent in those with relatively higher adiponectin levels [50].

This study has some limitations. Our genotyping of SNPs reported to be associated with adiponectin levels was limited to those in the $A D I P O Q$ gene (or their proxies, as indicated by $r^{2}>0.9$ ), but did not encompass SNPs outside it, either belonging (rs266717 and rs1426810) or not (rs4311394) to the $A D I P O Q$ locus. We cannot therefore exclude the possibility that these other SNPs may also play 
a role in modulating the common genetic background between HMW adiponectin and ACR. In addition, it is not known whether our data can be generalised to other populations with different environmental and/or genetic backgrounds; this aspect deserves further investigation.

In conclusion, our study shows a positive and independent correlation between HWM adiponectin and ACR. In addition, the $A D I P O Q$ gene accounts for a significant, although small, proportion of ACR heritability, as well as of the common genetic background shared by circulating HMW adiponectin and ACR, thus suggesting a causeeffect relationship between the two variables.

Acknowledgements This study was supported by Accordo Programma Quadro in Materia di Ricerca Scientifica nella Regione Puglia-PST 2006 and Italian Ministry of Health (RC2009 and RC2010) grants to C. Menzaghi.

Duality of interest The authors declare that there is no duality of interest associated with this manuscript.

\section{References}

1. Viberti GC, Hill RD, Jarrett RJ, Argyropoulos A, Mahmud U, Keen H (1982) Microalbuminuria as a predictor of clinical nephropathy in insulin-dependent diabetes mellitus. Lancet $1: 1430-1432$

2. Valmadrid CT, Klein R, Moss SE, Klein BE (2000) The risk of cardiovascular disease mortality associated with microalbuminuria and gross proteinuria in persons with older-onset diabetes mellitus. Arch Intern Med 160:1093-1100

3. Hillege HL, Fidler V, Diercks GF et al (2002) Urinary albumin excretion predicts cardiovascular and noncardiovascular mortality in general population. Circulation 106:1777-1782

4. Scherer PE (2006) Adipose tissue: from lipid storage compartment to endocrine organ. Diabetes 55:1537-1545

5. Schraw T, Wang ZV, Halberg N, Hawkins M, Scherer PE (2008) Plasma adiponectin complexes have distinct biochemical characteristics. Endocrinology 149:2270-2282

6. Kadowaki T, YamAUChi T (2005) Adiponectin and adiponectin receptors. Endocr Rev 26:439-451

7. Heidemann C, Sun Q, van Dam RM et al (2008) Total and highmolecular-weight adiponectin and resistin in relation to the risk for type 2 diabetes in women. Ann Intern Med 149:307-316

8. Spranger J, Kroke A, Mohlig M et al (2003) Adiponectin and protection against type 2 diabetes mellitus. Lancet 361:226-228

9. Weyer C, Funahashi T, Tanaka S et al (2001) Hypoadiponectinemia in obesity and type 2 diabetes: close association with insulin resistance and hyperinsulinemia. J Clin Endocrinol Metab $86: 1930-1935$

10. Chen J, Muntner P, Hamm LL et al (2004) The metabolic syndrome and chronic kidney disease in US adults. Ann Intern Med 140:167-174

11. De Cosmo S, Trevisan R, Minenna A et al (2006) Insulin resistance and the cluster of abnormalities related to the metabolic syndrome are associated with reduced glomerular filtration rate in patients with type 2 diabetes. Diabetes Care 29:432-434

12. Looker HC, Krakoff J, Funahashi T et al (2004) Adiponectin concentrations are influenced by renal function and diabetes duration in Pima Indians with type 2 diabetes. J Clin Endocrinol Metab 89:4010-4017

13. Hadjadj S, Aubert R, Fumeron F et al (2005) Increased plasma adiponectin concentrations are associated with microangiopathy in type 1 diabetic subjects. Diabetologia 48:1088-1092

14. Komaba H, Igaki N, Goto $\mathrm{S}$ et al (2006) Increased serum high molecular weight complex of adiponectin in type 2 diabetic patients with impaired renal function. Am J Nephrol 26:476-482

15. Leth H, Andersen KK, Frystyk J et al (2008) Elevated levels of high molecular weight adiponectin in type 1 diabetes. $\mathrm{J}$ Clin Endocrinol Metab 93:3186-3191

16. Jorsal A, Tarnow L, Frystyk J et al (2008) Serum adiponectin predicts all-cause mortality and end stage renal disease in patients with type I diabetes and diabetic nephropathy. Kidney Int 74:649-654

17. Saraheimo M, Forsblom C, Thorn L et al (2008) Serum adiponectin and progression of diabetic nephropathy in patients with type 1 diabetes. Diabetes Care 31:1165-1169

18. Jaziri R, Aubert R, Roussel R et al (2010) Association of ADIPOQ genetic variants and plasma adiponectin isoforms with the risk of incident renal events in type 2 diabetes. Nephrol Dial Transplant 25:2231-2237

19. von Eynatten M, Schneider JG, Hadziselimovic S et al (2005) Adipocytokines as a novel target for the anti-inflammatory effect of atorvastatin in patients with type 2 diabetes. Diabetes Care 28:754-755

20. von Eynatten M, Liu D, Bluemm A et al (2009) Changes in adiponectin multimer distribution in response to atorvastatin treatment in patients with type 2 diabetes. Clin Endocrinol (Oxf) 71:27-32

21. Strippoli GF, Navaneethan SD, Johnson DW et al (2008) Effects of statins in patients with chronic kidney disease: meta-analysis and meta-regression of randomised controlled trials. BMJ 336:645-651

22. Ruggenenti P, Cravedi P, Remuzzi G, Medscape (2010) The RAAS in the pathogenesis and treatment of diabetic nephropathy. Nat Rev Nephrol 6:319-330

23. Pajvani UB, Hawkins M, Combs TP et al (2004) Complex distribution, not absolute amount of adiponectin, correlates with thiazolidinedione-mediated improvement in insulin sensitivity. J Biol Chem 279:12152-12162

24. Sarafidis PA, Stafylas PC, Georgianos PI, Saratzis AN, Lasaridis AN (2010) Effect of thiazolidinediones on albuminuria and proteinuria in diabetes: a meta-analysis. Am J Kidney Dis $55: 835-847$

25. Menzaghi C, Trischitta V, Doria A (2007) Genetic influences of adiponectin on insulin resistance, type 2 diabetes, and cardiovascular disease. Diabetes 56:1198-1209

26. Hivert MF, Manning AK, McAteer JB et al (2008) Common variants in the adiponectin gene (ADIPOQ) associated with plasma adiponectin levels, type 2 diabetes, and diabetes-related quantitative traits: the Framingham Offspring Study. Diabetes 57:3353-3359

27. Richards JB, Waterworth D, O'Rahilly S et al (2009) A genomewide association study reveals variants in ARL15 that influence adiponectin levels. PLoS Genet 5:e1000768

28. Heid IM, Henneman P, Hicks A et al (2010) Clear detection of ADIPOQ locus as the major gene for plasma adiponectin: results of genome-wide association analyses including 4659 European individuals. Atherosclerosis 208:412-420

29. Tian C, Plenge RM, Ransom M et al (2008) Analysis and application of European genetic substructure using $300 \mathrm{~K}$ SNP information. PLoS Genet 4:e4

30. Menzaghi C, Ercolino T, Salvemini L et al (2004) Multigenic control of serum adiponectin levels: evidence for a role of the APM1 gene and a locus on 14q13. Physiol Genomics 19:170-174 
31. Menzaghi C, Coco A, Salvemini L et al (2006) Heritability of serum resistin and its genetic correlation with insulin resistancerelated features in nondiabetic Caucasians. J Clin Endocrinol Metab 91:2792-2795

32. Menzaghi C, Salvemini L, Paroni G et al (2010) Circulating high molecular weight adiponectin isoform is heritable and shares a common genetic background with insulin resistance in nondiabetic White Caucasians from Italy: evidence from a family-based study. J Intern Med 267:287-294

33. Placha G, Poznik GD, Dunn J et al (2006) A genome-wide linkage scan for genes controlling variation in renal function estimated by serum cystatin $\mathrm{C}$ levels in extended families with type 2 diabetes. Diabetes 55:3358-3365

34. Almasy L, Blangero J (1998) Multipoint quantitative-trait linkage analysis in general pedigrees. Am J Hum Gen 62:1198-1211

35. Didelez V, Sheehan N (2007) Mendelian randomization as an instrumental variable approach to causal inference. Stat Methods Med Res 16:309-330

36. Snijders TAB, Bosker R (1999) Multilevel analysis: an introduction to basic and advanced multilevel modeling. Sage, London

37. van Houwelingen HC, Arends LR, Stijnen T (2002) Advanced methods in meta-analysis: multivariate approach and metaregression. Stat Med 21:589-624

38. Tentolouris N, Doulgerakis D, Moyssakis I et al (2004) Plasma adiponectin concentrations in patients with chronic renal failure: relationship with metabolic risk factors and ischemic heart disease. Horm Metab Res 36:721-727

39. Tsioufis C, Dimitriadis K, Chatzis D et al (2005) Relation of microalbuminuria to adiponectin and augmented C-reactive protein levels in men with essential hypertension. Am J Cardiol 96:946-951

40. Sharma K, Ramachandrarao S, Qiu G et al (2008) Adiponectin regulates albuminuria and podocyte function in mice. J Clin Invest 118:1645-1656
41. Vionnet N, Tregouët D, Kazeem G et al (2006) Analysis of 14 candidate genes for diabetic nephropathy on chromosome $3 \mathrm{q}$ in European populations: strongest evidence for association with a variant in the promoter region of the adiponectin gene. Diabetes 55:3166-3174

42. Bouatia-Naji N, Meyre D, Lobbens S et al (2006) ACDC/ adiponectin polymorphisms are associated with severe childhood and adult obesity. Diabetes 55:545-550

43. Laumen H, Saningong AD, Heid IM et al (2009) Functional characterization of promoter variants of the adiponectin gene complemented by epidemiological data. Diabetes 58:984-991

44. Morris NJ, Gray-McGuire C, Stein CM (2009) Mendelian randomization in family data. BMC Proc 3(Suppl 7):S45

45. Krolewski AS, Poznik GD, Placha G et al (2006) A genome-wide linkage scan for genes controlling variation in urinary albumin excretion in type II diabetes. Kidney Int 69:129-136

46. Mottl AK, Vupputuri S, Cole SA et al (2009) Linkage analysis of albuminuria. J Am Soc Nephrol 20:1597-1606

47. Gu HF, Alvarsson A, Efendic S, Brismar K (2009) SOX2 has gender-specific genetic effects on diabetic nephropathy in samples from patients with type 1 diabetes mellitus in the GoKinD study. Gend Med 6:555-564

48. Beige J, Heipmann K, Stumvoll M, Körner A, Kratzsch J (2009) Paradoxical role for adiponectin in chronic renal diseases? An example of reverse epidemiology. Expert Opin Ther Targets 13:163-173

49. Zoccali C, Mallamaci F, Tripepi G et al (2002) Adiponectin, metabolic risk factors, and cardiovascular events among patients with end-stage renal disease. J Am Soc Nephrol 13:134-141

50. Zoccali C, Mallamaci F, Tripepi G (2004) Inflammatory proteins as predictors of cardiovascular disease in patients with end-stage renal disease. Nephrol Dial Transplant 19(Suppl 5): V67-V72 